\title{
An Augmented Method For Finding Character Lines From a Gray Scene Image
}

\author{
( シーン内の文字列領域の改良した抽出方法)
}

\author{
Yongmei $\mathrm{Liu}^{\dagger 1}$, \\ Tsuyoshi Yamamura $^{\dagger 2}$, Toshimitsu Tanaka ${ }^{\dagger 3}$, \\ Noboru Ohnishi (member) ${ }^{\dagger 1, \dagger 4}$
}

\begin{abstract}
An augmented method for finding character lines in a gray scene image is proposed. In the proposed approach, we use several heuristics of both characters (such as size, symmetry of pixels and bimodality of intensity histogram) and character lines (such as proximity of characters and alignment of arrangement) to discriminate characters from other objects in a scene image. Experimental results indicated that the method performed well for signboards imaged from a relatively wide range of viewing directions.
\end{abstract}

Key words: Character Detection, Character Arrangement, Angled View

\section{Introduction}

Character information plays an important role in our everyday life. If characters in both scene images and document images could be extracted and recognized by a computer system, this information would be applied to a number of applications such as vision-based mobile robot navigation and image database indexing. In our previous research, we developed a method for finding characters in a gray scene image ${ }^{1)}$. In the method, first, we extract subregions with high spatial frequency and great variance in gray level from an input scene image as candidates of character components. Then, we select characters by using several heuristics of characters and character lines. Experiments on 60 images show that more than $80 \%$ of character lines in both indoor and outdoor scene images are detected successfully. However, in real applications, characters may be imaged from a nonorthogonal viewing direction. When we applied the proposed method to this kind of images,

Received December 1, 2000; Revised May 16, 2001; Accepted June 7, 2001

$† 1$ Bio-Mimetic Control Research Center, The Institute of Physical and Chemical Research (Riken)

(2271-130, Anagahora, Shimoshidami, Moriyama-ku, Nagoya-shi, Aichi 463-0003, Japan )

$† 2$ Aichi Prefectural University

( Nagakute-Cho, Aichi-Gun, Aichi, 480-1198, Japan)

†3 Meijo University

( 1-501, Shiogamaguchi, Tenpaku-ku, Nagoya-shi, Aichi, 468-8502 Japan )

† Nagoya University

( Furo-cho, Chikusa-ku, Nagoya-shi, Aichi, 464-8703, Japan ) the extraction rate dropped to about $40 \%$. This is because when a signboard is viewed at an angle, some wellseparated characters appear as connected components, and also characters in the same line no longer have the features of similarity in size and horizontal (vertical) alignment. Therefore, we improved our approach by adding three new heuristics of characters and removing the restriction of aspect ratio that is only applicable to characters viewed from the front. We also modified the threshold value of each heuristic in order to make it more appropriate to characters imaged from a wide range of viewing directions.

\section{Method for Finding Characters from a Scene Image}

We are aiming at applying our results to a characterbased mobile robot navigation system. Considering the computation time, here, we only use the heuristics of characters and character arrangement to identify characters in a scene image. There are a number of related ideas reported in literatures ${ }^{2) 3}$. However, some features used in those methods cannot be applied to Chinese and Japanese characters. The method presented here has been primarily designed to identify Chinese and Japanese characters written on signboards and traffic (road) signs, and the characters may be imaged from a relatively wide range of viewing directions. Signboards with special art design and characters that touch with background noise, such as trees, frames of sign- 
boards are not considered as our targets.

First, we change an input image into a binary image using thresholding. By calculating the minimum and maximum values of $\mathrm{x}$ and $\mathrm{y}$ coordinates of every "white" and "black" object, we can obtain the bounding rectangle of each object. Many Chinese and Japanese characters are composed of more than one part, considering these multi-segment characters, we then combine nearby bounding boxes with consideration of their positional relationships ${ }^{1)}$. Then we use the following five restrictions to remove non-character components.

I Size. The width and height of a bounding rectangle.

II Area. The number of pixels in a bounding rectangle.

Size and area parameters are used to eliminate very large objects (walls and buildings in a scene image) and very small objects (noise). Rectangles that satisfy Eqs.(1), (2) and (3) are considered as possible character regions.

$$
\begin{aligned}
& \min (\text { width }, \text { height })>13 \text { pixels } \\
& \max (\text { width }, \text { height })<120 \text { pixels } \\
& 280<\text { area }<7950
\end{aligned}
$$

III Density.

We use the ratio of the number of black (white) pixels to white (black) pixels in the bounding rectangle of each object to discriminate characters and noncharacters. We choose rectangles with a density ratio larger than 0.14 as possible characters.

IV Symmetry of pixels.

The structures of most Japanese (Chinese) characters are balanced. We use the ratio between the number of black (white) pixels in the upper (left) and lower (right) parts in a bounding rectangle as a restriction to separate characters from other objects. Figure 1 shows some examples. For characters, we restrict the ratio to be larger than 0.510 and less than 1.96 .

V Bimodality of intensity histogram.

In the bounding rectangles, the gray-level contrast between characters and their backgrounds

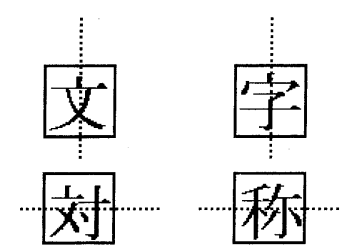

Fig. 1 Symmetry of pixels in a character bounding rectangle

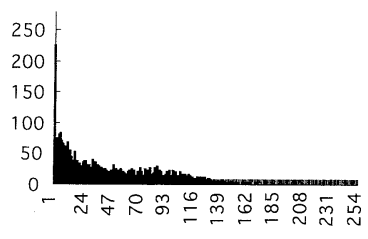

(a) non-character region (leaves of tree)

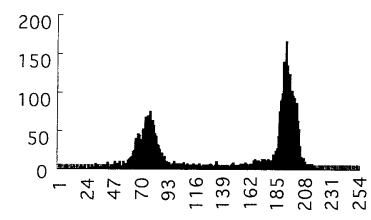

(b) character region
Fig. 2 Bimodality of intensity histogram.

is relatively high, and the gray-level histograms of character regions have two distinct peaks (bimodality). We compute the gray-level histogram of each bounding rectangle and examine the bimodality of the histogram. Then we use the gray level difference between the two peaks as a threshold to select characters. Rectangles which satisfy the condition that $\mid$ Peak1 - Peak2 $\mid>60$ are selected as possible characters. Examples of both character and non-character histograms are shown in Fig.2.

Here, features II to IV are supplemented heuristics. We introduced a cost function to decide the threshold value of each feature. The cost function is defined as

$$
C F=W_{f p} N_{f p}+W_{f n} N_{f n},
$$

where $f p$ (false positive) represents the case in which noncharacters are misdetected, and $f n$ (false negative) represents the case in which characters are not detected. $W_{f p}, W_{f n}$, and $N_{f p}, N_{f n}$ are the weights and numbers of each case, respectively. Here, we set $W_{f p}=1$ and $W_{f n}=10$. The reason that we let the value of $W_{f n}$ be 10 times of $W_{f p}$ is because we use five restrictions to discriminate characters and noncharacters. If a noncharacter is misdetected by one restriction, it still can be rejected based on the other four features and restrictions on character lines. On the other hand, if a character is mis-deleted, it may never be recovered in the following steps. In order to decide the threshold values, first, we selected a set of sample images (40) including both indoor and outdoor scenes, as well as images viewed at an angle. We labeled each bounding rectangle in these images manually as a character or a noncharacter. Then, for each feature, using the set of images, we applied a range of values as thresholds to calculate the cost function; the value that minimizes the cost function will be selected as a real threshold for the feature. we extract subregions with high spatial frequency and great variance in grey-level from an input scene image as candidates of character components.

Figure 4(b) shows some examples of the charac- 
ter extraction results using the above five constraints. Characters are correctly extracted, however, some noncharacter regions also remained. Without character recognition capabilities, it is difficult to discriminate characters from other objects based simply on the features of character itself. A character line that consists of several characters can also provide additional information for this classification. Here, we use the following arrangement features of a character line.

I Similar height (width) of neighboring components

Instead of using restriction on size, we only restrict the difference in height (for originally horizontal character lines) or width (for originally vertical character lines) between two neighboring characters in the same line to less than $\frac{1}{3}$ of their average height (width).

II Proximity of characters

We limit the space between two adjacent characters in the same character line by the following equation:

$$
c<3 \times \min (a, b),
$$

where $a, b$ are the width of each rectangle, and $c$ is the space between two rectangles (Fig.3(a)).

III Alignment of arrangement

For a straight character line viewed at an angle, sometimes it will not be an approximately vertical or horizontal line in the image plane, it may be a slanting line. Therefore we relaxed the restriction on alignment. Here, take a originally horizontal character line as an example. For two neighbor bounding rectangles $I$ and $I I$, if $\mathrm{d}>0$, rectangles $I$ and $I I$ are considered as related.

$$
d=\min \left\{x_{u_{I}}, x_{u_{I I}}\right\}-\max \left\{x_{l_{I}}, x_{l_{I I}}\right\},
$$

where $x_{u}, x_{l}$ are $\mathrm{x}$-coordinates of the upper and lower edge of a rectangle. For a chain of related bounding rectangles, if they satisfy Eq.(7), they are judged as aligned. This restriction is use to select rectangle chains in which the curve that goes through the center of each component is an upward (Fig.3(i)) or a downward curve (Fig.3(ii)), and filter out an up and down curve (Fig.3(iii)). It is similar for a vertical line.

$$
\sum_{i=0}^{n-1}\left|x_{c_{i}}-x_{c_{i+1}}\right|-\left|\sum_{i=0}^{n-1}\left(x_{c_{i}}-x_{c_{i+1}}\right)\right| \leqq 15,(7)
$$

where $x_{c}$ is the x-coordinate of each rectangle's center, $i$ and $i+1$ are two neighboring rectangles, and $n$ is the number of objects in the rectangle chain.

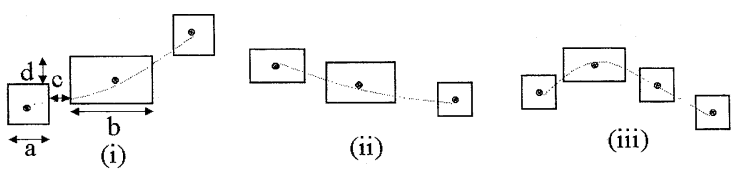

Fig. 3 Character line arrangement.

Table 1 Experimental Results on Signboards Viewed from the Front (60 images).

\begin{tabular}{l|c|c|c|c}
\hline \hline & $\begin{array}{c}\text { Number of } \\
\text { Char. Line }\end{array}$ & $\begin{array}{c}\text { Number of } \\
\text { Extractions }\end{array}$ & $\begin{array}{c}\text { Rate of } \\
\text { Extraction }\end{array}$ & Error \\
\hline Method 1 & 77 & $63(+8)$ & $81.8 \%(92.2 \%)$ & 12 \\
Method 2 & 77 & $65(+7)$ & $84.4 \%(93.5 \%)$ & 9 \\
\hline
\end{tabular}

Table 2 Results on Signboards Viewed at an Angle (70 images).

\begin{tabular}{l|c|c|c|c}
\hline \hline & $\begin{array}{c}\text { Number of } \\
\text { Char. Line }\end{array}$ & $\begin{array}{c}\text { Number of } \\
\text { Extractions }\end{array}$ & $\begin{array}{c}\text { Rate of } \\
\text { Extraction }\end{array}$ & Error \\
\hline Method1 & 84 & $37(+10)$ & $44.0 \%(56.0 \%)$ & 8 \\
Method2 & 84 & $67(+12)$ & $79.8 \%(94.1 \%)$ & 12 \\
\hline
\end{tabular}

\section{Experimental Results and Discussion}

We conducted experiments using the same 40 outdoor and 20 indoor scene images viewed from the front (Fig.4(1) shows an example) as in ${ }^{1)}$. We also added 70 scene images in which signboards are viewed at an angle. All input images were digitized into 256 gray levels and $640 \times 480$ pixels. Among the 70 new images, 31 images were generated by image transformation. Using images that viewed from the front, we created images of signboards (Fig.4(2) shows an example) that observed at viewing directions of $\theta=20^{\circ}$ (16 images) and $45^{\circ}$ (15 images)(around $\mathrm{X}$-axis or $\mathrm{Y}$-axis). The other 39 images are real scene images (Fig.4(3)(4) are two examples) taken by us, and the exact viewing angles of the camera are unknown. Figure $\mathbf{4}$ shows some experimental results. In the figure, (a) input images, (b) the results obtained using only features of characters and (c) the results obtained using both features of characters and character lines.

Tables 1 and $\mathbf{2}$ list the results obtained using two methods. In the tables, "Method1" represents the method in the previous work ${ }^{1)}$ and "Method2" represents the method proposed in this paper; "Error" indicates the number of noncharacter lines that were misextracted, and values in parentheses represent the numbers (rates) of character lines, the characters of which are partially extracted. For signboards viewed from the front, the extraction rates of the two methods are almost the same. However, for signboards imaged from an angle, the result of the new approach is much better than that of the old one, indicating the effectiveness of 
the improved method. The processing time differs from image to image. Using the method in ${ }^{1)}$, it generally takes 15 to 20 seconds to process an image on a Sun Ultra 1 Workstation, while it requires only 5 to 8 seconds using the improved method. This makes our new approach more applicable to robot navigation tasks.

In the experiments, the threshold values for discriminating characters are decided based on a cost function (as described in section 2). The threshold values for restriction $I, I I$ and $I I I$ on character lines are selected based on experiments on 30 sample images including 35 character lines. These samples contain character lines with various size, style, lengths, directions and viewing angles. The threshold values are adjusted little by little according to the experimental results on these images, and the values with the best results are selected as the final thresholds. Using the selected thresholds, character line extraction rate on these 30 sample images was $91.43 \%$ (including partially detected character lines). We then applied these thresholds to all the 130 images, and the extraction rate was $93.78 \%$, and this showed the appropriateness of the selected values.

The proposed method could detect most of the character lines in these 130 scene images. Several special characters such as "1"," - " and “三” do not satisfy the five restrictions that we used to select character regions, we missed to extract them at the first stage. However, if they are in the middle of a character line, in many cases, this kind of false negative can be recovered. The "-”" in Fig.4(1) shows an example of this problem. However, we failed in finding characters of low gray-level contrast and characters that touch with background noise. The last character in Fig.4(1) touches with the frame, we failed to separate it from the background. In Fig.4(4), we found the characters on the bus stop successfully, however, we failed to extract characters on the background building because some of the characters are partly obscured by the leaves of trees. "Method1" also failed on most of the images failed by "Method2", however, the performance of "Method2" is much better than "Method1", especially for characters viewed at an angle.

\section{Conclusion}

In this paper, we proposed an augmented method for finding character lines in a gray scene image. Experimental results indicate that our method performed well on characters taken from a relatively wide range of viewing directions. Once a signboard is identified, using the (a)
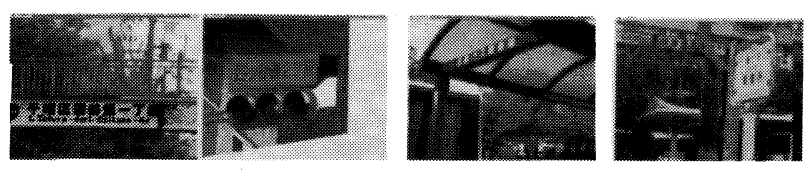

(b)

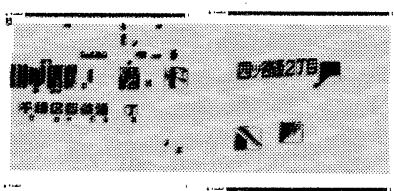

(c)

母吩通2T目

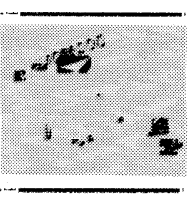

10

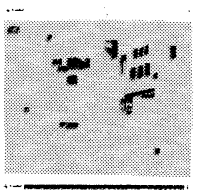

ili

(4)

Fig. 4 Experimental Results

information of the shape of the signboard, we can also estimate the camera viewing angle and rectify the distorted signboard image ${ }^{4)}$.

\section{[References〕}

1) Y. Liu, T. Yamamura, N. Ohnishi and N. Sugie, "Extraction of Character String Regions from a Scene Image," Trans. IEICE Japan, J 81-D-II, 4, pp. 641-650 (1998 In Japanese).

2) A. K. Jain and B. Yu, "Automatic Text Location in Images and Video Frames," Pattern Recognition, 31, pp. 2055-2076 (1998)

3) S. Messelodi and C. M. Modena, "Automatic Identification and Skew Estimation of Text Lines in Real Scene Images," Pattern Recognition, 32, pp. 791-810 (1999)

4) Y. Liu, T. Yamamura, T. Tanaka, and N. Ohnishi, "Extraction and Distortion Rectification of Signboards in a Scene Image for Robot Navigation," Trans. IEEJ, 120-C, 7, pp. 1026-1034 (2000)

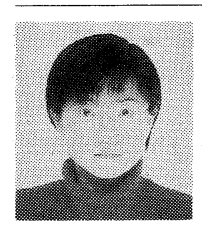

Yongmei Liu She received the B.E.degree in computer science and engineering from Beijing University of Aeronautics and Astronautics, Beijing, China, in 1990, and M.E. degree in information engineering from Nagoya University, 1996. She is currently a technical staff at Bio-Mimetic Control Research Center, RIKEN. She is a member of IEEE, IEICE and RSJ.

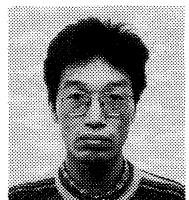

Tsuyoshi Yamamura $\mathrm{He}$ received his $\mathrm{BE}, \mathrm{ME}$ and Ph.D degrees from Nagoya University in 1987, 1989 and 1994, respectively. He is presently an Associate Professor in the Faculty of Information Science and Technology, Aichi Prefectural University. He is engaged in research on Natural Language Processing and Visual Information Processing. He is a member of IEEE, IEICE and IPSJ.

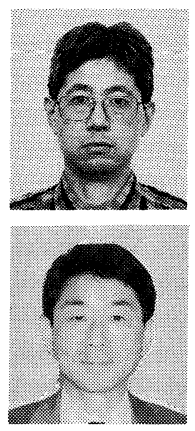

Toshimitsu Tanaka He received his BE, $\mathrm{ME}$ and Ph.D Degrees from Nagoya University in 1982,1984 and 1992, respectively. He is presently a professor in Meijo University. He is engaged in research on computer graphics. Dr. Tanaka is a member of IPSJ and IEICE.

Noboru Ohnishi He received the B. Eng., M. Eng. and D. Eng. degrees from Nagoya University, Nagoya, Japan, in 1973, 1975 and 1984, respectively. He is a Professor in the Department of Information Engineering, and concurrently a Head of Laboratory for Bio-mimetic Sensory System at the Bio-mimetic Control Research Center of RIKEN. His research interests include computer-vision and -audition, robotics, bio-cybernetics, and rehabilitation engineering. Dr. Ohnishi is a member of IEEE, IEEJ, IEICE, IPSJ, SICE, JNNS, IIITE and RSJ. 